\title{
Delaying Thermalization in a Periodically Driven System
}

\section{Researchers have kept a Floquet system in a prethermal state for a record length of time.}

\section{By Marric Stephens}

$\square$ eriodically driven systems provide a platform for investigating quantum phenomena and exotic states of matter (see Viewpoint: Time Crystals in Open Systems). These phenomena are easiest to study when these so-called Floquet systems are in a nonequilibrium "prethermal" regime. But eventually, the energy delivered by the periodic driver distributes through the system, heating it to equilibrium and washing away information about those interesting quantum states. Now, in a Floquet system based on nuclear spins surrounding a diamond defect, William Beatrez, at the University of California, Berkeley, and colleagues have managed to stave off thermalization for the longest time yet [1]. The achievement widens the window through which physicists can observe prethermal and thermalization dynamics and could lead to high-sensitivity magnetometers and spin sensors.

To generate a Floquet state, the researchers polarized a diamond nitrogen-vacancy center by pumping it with a laser. They then used microwaves to transfer this polarization to surrounding carbon-13 nuclei, which spread the polarization

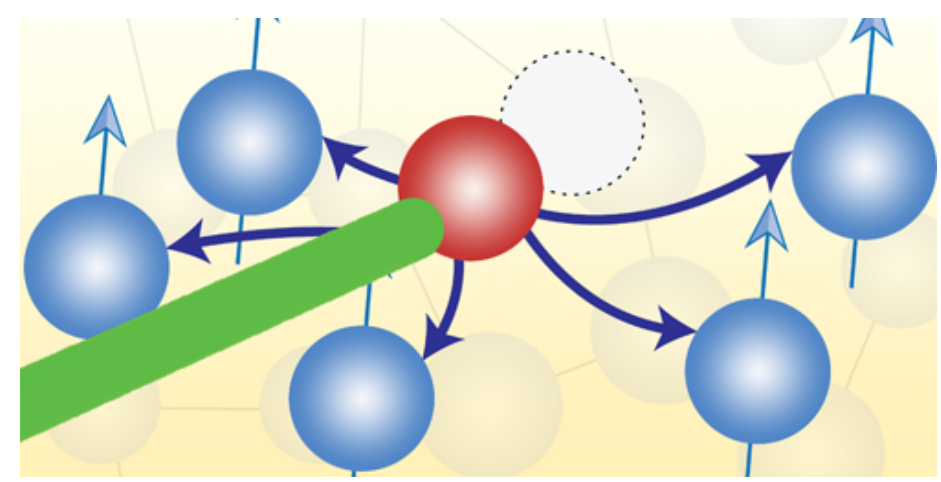

Credit: W. Beatrez et al. [1] throughout the rest of the diamond via dipole-dipole coupling. Left alone, this state decays in around $1.5 \mathrm{~ms}$. But by driving the system with radio-frequency pulses, the researchers induced a collective precession in the nuclear spins, prolonging the polarization lifetime more than 60,000 -fold. They measured this precession using nuclear magnetic resonance.

Beatrez and colleagues say that the degree to which their technique extends the prethermal period is surprising. Previous experiments using ultracold atoms sustained the prethermal state for around 22,000 driving cycles; in the new experiment, it lasted for 1.6 million cycles. The team hopes that they can use this longer view to study nonequilibrium phenomena, such as time-crystalline behavior.

Marric Stephens is a Corresponding Editor for Physics based in Bristol, UK.

\section{REFERENCES}

1. W. Beatrez et al., "Floquet prethermalization with lifetime exceeding $90 \mathrm{~s}$ in a bulk hyperpolarized solid," Phys. Rev. Lett. 127,170603 (2021). 